\title{
Michel Butor : des profondeurs ${ }^{1}$
}

\author{
Roger-Michel Allemand \\ Laboratoire Babel - Université de Toulon
}

Fin juillet 2016, un journaliste de Reporters sans frontières me demande le numéro de téléphone de Michel, pour un dossier sur le jazz. Michel est fatigué, je ne le donne pas.

Nuit du 24 au 25 août, la nouvelle tombe. France Culture m'appelle et m'envoie un courriel urgent, pour deux émissions le jour même. Je refuse, d'autres se presseront. Je décline toutes les invitations à rendre hommage. Question de respect.

\footnotetext{
${ }^{1}$ Ce texte est extrait de la rencontre organisée dans le cycle Impromptu au Petit Palais, à Paris, le jeudi 12 novembre 2009, et diffusée en direct sur France Culture puis podcastée dans Les Chemins de la connaissance, à l'occasion de la parution de Allemand et Butor, 2009.
} 
Messages de sympathie. Éric Tanguy me dit que, paraît-il, Michel a écrit sur sa musique et apprend que c'est moi qui la lui ai fait découvrir, en souvenir de nos années de lycée2. Condoléances.

6 novembre, je parle en ami avec l'autre grand Michel, Deguy, qui a cette formule: "Nous sommes dans le deuil de Butor. "Évocations du Petit Palais, Forence Trocmé m'offre l'enregistrement.

Les voici donc, ces échanges d'alors, ressurgis du passé pour en marquer le trait définitif. J'en avais oublié la teneur, je l'avais toujours sue. La mort. Elle était spectre et son génie.

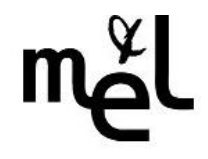

Jeudi 12

\section{novembre}

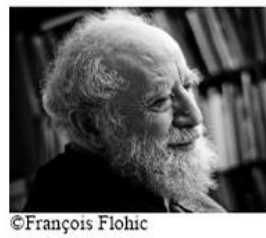

de $18 \mathrm{~h}$ à $19 \mathrm{~h} 30$

à l'auditorium du Petit Palais, musée des BeauxArts de la Ville de Paris (entrée libre et gratuite dans la limite des places disponibles)

Avenue Winston Churchill 75008 métro Ch-Elysées Clémenceau

\section{Rencontre avec l'écrivain}

\section{Michel Butor}

\section{présenté par Roger-Michel Allemand}

La Maison des écrivains et de la litterature et Argol éditions organisent cet entretien avec Michel Butor à l'occasion de la parution du livre : Michel Butor/Rencontre avec RogerMichel Allemand, collection «Les Singuliers », Argol éditions. Avec une projection du film Michel Butor, á l'écart (30'), réalisé par François Flohic, documentariste et photographe, production Argol.

Michel Butor, né en 1926, a poursuivi une carrière universitaire aux États-Unis, en France, puis à Genève, jusqu'en 1991. Il est l'auteur d'un grand nombre de livres fondamentaux, tel La Modiffcation, publié en 1957 aux éd. de Minuit. En 2006, il a commencé la publication de ses ceuvres complètes en dix volumes aux éditions de la Différence.

Roger-Michel Allemand, Docteur ès lettres en littérature contemporaine, a publié de nombreux ouvrages autour du nouveau roman.

Roger-Michel Allemand - Il y a cette question qui vous a été posée et qui est très intéressante : « Est-ce que Butor est un bon

2 J'avais fait de même pour les compositions de mon vieil ami Aldo Romano. 
écrivain? » Vous n'avez pas répondu, mais j'ai un début de réponse, tiré de notre livre d'entretiens, où vous disiez qu'un "bon photographe est celui qui se fond dans le paysage " (Allemand et Butor, 2009, p. 19), c'est celui qui disparaît, qui s'efface (voir Allemand, 2011). Or, justement, vous êtes à l'écart, depuis fort longtemps à l'extérieur des cercles parisiens littéraires, et j'aimerais que vous reveniez sur cette idée d'effacement, de discrétion. En quoi vous est-elle nécessaire pour produire votre œuvre?

Michel Butor - Eh bien, je n'ai pas envie de parler de moi, j'ai envie de parler des autres, des choses, de la réalité, etc., mais dans certains cas, je suis obligé de parler de moi, parce que je suis obligé de préciser le point de vue que j'ai. Je suis obligé d'expliquer que si je vois les choses de cette façon, c'est parce que je suis allé dans tel pays, par exemple, à tel moment, qu'il m'est arrivé ceci ou cela. C'est ce qui fait que dans mes livres, il y a ${ }^{3}$ des fragments d'autobiographie, tout le temps, mais ils sont faits pour aider à voir autre chose que moi. Je suis dans l'image pour que l'on puisse préciser la triangulation, si vous voulez. Ces fragments d'autobiographie ont incité un certain nombre de gens à me demander de compléter, en me posant des questions dans des entretiens. Je pense au livre d'André Clavel qui s'intitule Curriculum vitæ (Clavel et Butor, 1996). Donc j'ai raconté à peu près ce qu'on voulait que je raconte, mais il y a toujours des détails qui peuvent venir en plus, alors évidemment, ça n'en finit pas: on peut me poser des questions encore et, en général, j'essaie de répondre.

3 La tournure reviendra plus de trente fois dans ces échanges; elle est davantage qu'un tic de langage (voir Allemand et Butor, 2009, p. 71-72, et Allemand, 2012). 
Cela pour dire que je n'écris pas du tout pour me cacher, ce n'est pas du tout mon idée. J'écris en partie pour m'effacer, pour faire voir le reste, mais c'est ce qui se passe aussi chez tous les grands autobiographes. Jean-Jacques Rousseau n'écrit pas Les Confessions (2009) pour parler de lui. Il parle de lui parce que c'est indispensable pour que l'on comprenne sa situation à l'intérieur de cette société dont il veut parler et qu'il veut dévoiler. Il y a des écrivains plus ou moins narcissiques, mais la plupart du temps, contrairement à une idée répandue, l'écrivain ne l'est pas ; il est très inquiet de ce qu'il écrit. "Est-ce que vous êtes un bon écrivain? » Ça, c'est une question à laquelle on ne peut pas répondre. Il faut absolument que ce soit les autres qui le fassent. Si l'on essaie de répondre soi-même, on se trompe. On a donc besoin du discours et du regard des autres pour cela.

C'est un peu comme ma relation avec la poésie. Quand j'étais jeune, au lycée, à l'université, j'ai écrit beaucoup de poésie, et puis, quand je me suis mis à écrire des romans, je me suis interdit d'écrire de la poésie, pour que toute ma force poétique puisse passer par le roman, mais au bout d'un certain temps, ç'a explosé et je me suis mis à écrire des livres avec des artistes et à écrire des textes qui pouvaient convenir à ce que faisaient les artistes à cette époque-là. Est-ce que c'était de la poésie ? Eh bien, je ne pouvais pas répondre à ce moment-là. Il fallait absolument que les autres me disent que c'était de la poésie. Je le désirais de tout mon cœur mais je ne pouvais pas le dire moi-même. Vous savez, j'ai souvent cité cette phrase de Chesterton: "Il y a trois vocations qui ne peuvent pas se désigner elles-mêmes : le saint, le sage et le poète. » Si un moine dit qu'il est un saint, c'est qu'il ne l'est pas. Si quelqu'un dit : « je suis un sage », c'est qu'il ne l'est pas encore. De même, un poète qui dit qu'il est un poète, c'est qu'il y a quelque chose qu'il n'a pas compris (voir Allemand et Butor, 2009, p. 32). 
Ce sont toujours les autres qui doivent le dire. Alors ce sont les autres qui peuvent dire si je suis un bon écrivain.

R.-M. A. - Et la réponse me semble assez évidente. En ce qui concerne le travail de l'écriture, vous dites souvent que ce qui vous anime, c'est la surprise de découvrir ce qui vient : " aussi poussée que soit la programmation, [l'imprévu] est toujours là. Le texte s'écrit sous mes doigts et ne me satisfait que s'il me surprend. [...] Il m'échappe, et c'est en cela qu'il est mien, qu'il me rend moi-même. » (Allemand et Butor, 2009, p. 123) Cette écriture qui échappe au contrôle de l'écrivain, c'est un peu paradoxal : est-ce que cela voudrait dire que l'inspiration vous transcende? Vous traverse (voir ibid., p. 129)?

M. B. - Me transcende, je ne sais pas. Me traverse, certainement. Oui, il y a quelque chose qui me traverse et c'est ça qui me fait vivre. J'écris parce qu'il y a des choses que je ne sais pas comment dire. C'est pour cela que j'écris. Il y a tout le temps des choses que nous ne savons pas nommer, que nous ne savons pas expliquer, que nous ne savons pas décrire. Prenons les crises économiques: on croit qu'on est capable d'en parler, mais en réalité, nous n'en sommes pas encore capables. Les théories économiques actuelles sont encore dans le balbutiement et il faut inventer quelque chose de profondément nouveau. Il y a un certain nombre de phénomènes qu'il faut nommer, qu'il faut devenir capables de nommer et donc auxquels il faut donner des noms différents de ceux avec lesquels on les nommait jusqu'à présent. Cela est vrai pour toutes sortes de domaines. Il y a des choses dont nous ne sommes pas capables de parler et pourtant nous sentons qu'il est indispensable de le faire. Cela demande un effort gigantesque de réussir à parler de ce dont on ne pouvait pas parler. C'est pourquoi la plupart des 
gens y renoncent. Ils n'arrivent pas à le faire. Il y a quelques artistes, quelques écrivains qui réussissent à poursuivre cela et, évidemment, ils perturbent l'ensemble. Il y a, disons, un langage qui essaie de cacher ces lacunes, et puis il y a quelqu'un qui se met à déchirer les voiles, comme ça. Si vous voulez, on met des pansements... Nous sommes un corps tout déchiré de blessures ; alors on met des pansements dessus et on arrive à l'oublier un peu. Puis il y a quelqu'un qui se met à arracher ces pansements et qui dit : « Il ne faut pas seulement panser cela, il faut le guérir. » Ce qui est quelque chose de complètement différent. On comprend la résistance que cela peut provoquer.

R.-M. A. - Quelles sont les blessures que vous avez mises au jour, justement?

M. B. - Ah... J'ai essayé de mettre des blessures au jour, et je ne peux pas le dire moi-même. J'ai absolument besoin que d'autres disent que j'ai réussi à montrer ceci ou cela. Moi, je suis dans un effort qui est de toute façon interminable. Je ne peux pas dire si j'ai réussi ou pas. D'ailleurs, je ne peux pas dire si mes livres ou si mes textes sont réussis ou pas. J'ai travaillé sur mes textes, je les ai corrigés, jusqu'à un moment où je ne pouvais plus aller plus loin. Peut-être qu'à certains moments, j'aurais dû aller plus loin encore, mais je n'y ai pas réussi, parce que les circonstances, la fatigue, etc. ont fait que j'ai pu mener ce texte jusqu'à ce point mais que je n'ai pas pu aller plus loin. C'est alors que je le propose aux autres. Pour qu'ils puissent, eux, aller plus loin. Est-ce que le texte est réussi ? Est-ce que le livre est réussi ou pas? Je ne peux pas le dire moimême. Je le publie à partir du moment où je n'arrive plus à le pousser plus loin. Cela est vrai pour les romans, bien sûr, j'ai éprouvé ça très fortement, mais c'est vrai même pour des poèmes 
de trois lignes. Autrefois j'étais tout à fait incapable d'écrire des poèmes de trois lignes. Il m'a fallu soixante ans d'efforts pour y parvenir, mais même les poèmes de trois lignes, je ne peux pas dire qu'ils sont réussis. Je peux dire seulement : «Voilà, je n’arrive pas à aller plus loin que ça. »

R.-M. A. - C'est un point commun avec Hokusai, qui, vers la fin de sa vie, disait qu'à partir de cent ans, il commencerait peutêtre à devenir un bon peintre.

M. B. - Mais je ne renonce pas! Je ne renonce pas à devenir un écrivain intéressant. J'ai déjà beaucoup de choses derrière moi, mais peut-être qu'à quatre-vingt-dix ans, j'écrirai quelque chose qui vaudra la peine qu'on s'en souvienne ${ }^{4}$.

R.-M. A. - «Il s'agit de disparaître, non plus dans le malheur, mais dans le bonheur d'autrui." (Allemand et Butor, 2009, p. 171) Est-ce que vous auriez la gentillesse de revenir sur cette déclaration de vous?

M. B. - J'ai dit que l'écriture était une forme positive du suicide (voir ibid., p. 169-171). Ceci parce qu'on se met à écrire parce qu'on a des problèmes. On est dans une société dont on sent qu'elle ne marche pas et on sent qu'à l'intérieur de cette société, on est soi-même un problème. On ne sait pas qui l'on est, on ne sait pas ce qu'on pourrait faire, on ne sait pas ce qu'il faudrait faire, et ainsi de suite. Il y a une espèce d'incompatibilité entre un individu et la société qui l'entoure. Quelquefois, cet individu estime que la seule solution à cette antinomie, à ce malheur

4 Décédé le 24 août 2016, Michel Butor n'a pas atteint ses quatre-vingt-dix ans, qu'il aurait eus le 14 septembre. 
d'opposition, à ce duel entre lui et les autres, c'est qu'il se supprime. À partir de ce moment-là, il y a une certaine guérison, si vous voulez, mais le problème va se reposer exactement de la même façon avec d'autres, puisque les circonstances sont telles, les conditions sont telles que cela a produit un personnage de ce genre et que cela en produira d'autres.

Il est donc plus intéressant d'assumer ce malheur collectif, ce malheur de plusieurs individus. Au lieu de se supprimer soimême, le mieux, c'est d'essayer de changer le reste. Cette incompatibilité, je vais essayer de la changer en transformant la réalité autour de moi, de toutes sortes de façons: en construisant des maisons par exemple, ou en faisant des peintures qui feront que je verrai la réalité autrement. Je peux transformer la réalité autour de moi en écrivant des livres dans lesquels il y a des inventions stylistiques, littéraires, etc. qui feront qu'on pourra parler - pour revenir à ce que nous disions tout à l'heure - qu'on pourra enfin parler de ce dont on ne pouvait pas parler auparavant. À partir de ce moment-là, donc, on supprime la différence que l'on était. Vous voyez en quoi cela reste un suicide, avec le thème de l'effacement : on s'efforce de supprimer la différence que l'on était, mais au lieu de supprimer simplement le côté individuel de la différence, on s'efforce de la supprimer dans son ensemble. Par conséquent, on va s'effacer mais dans la transformation de l'ensemble et le malheur de l'un devient le bonheur des autres. Évidemment, c'est une tâche qui est inépuisable.

La société de la fin du XIX ${ }^{\text {e }}$ siècle, en France, produit un individu comme Rimbaud et Rimbaud s'efforce d'arranger ça, parce que c'est une situation impossible d'être Rimbaud à Charleville. On peut très bien imaginer, à un certain moment, Rimbaud se suicidant et nous ne connaîtrions rien de son œuvre. Évidem- 
ment, le voyage au Harrar, c'est une espèce d'équivalent au suicide et c'est une forme d'effacement disons exemplaire. Heureusement, Verlaine a publié Rimbaud, et ce fait empêche que le drame de Rimbaud recommence de façon stupide, dans une répétition infernale. Alors, il y aura d'autres problèmes, qui produiront d'autres individus, mais il faut espérer que ces individus, qui sont des incarnations des problèmes de la société, soient suffisamment doués d'abord, et puis aient suffisamment de chance, dans leur entourage, dans les circonstances, pour qu'ils réussissent à opérer cette transformation. Cette transformation, pour moi, on l'opère avant tout par le langage, mais je ne détache pas du tout le langage des autres activités de l'esprit et des autres activités humaines, quelles qu'elles soient. J'essaie d'opérer cette révolution par le langage d'une façon aussi douce que possible, en évitant toute violence inutile. Il y a trop de violence inévitable. Donc j'essaie d'opérer cette rénovation du langage, mais j'appelle à mon aide tout le reste : les peintres, les musiciens, les cuisiniers et même les sportifs.

R.-M. A. - Il était impossible d'être Rimbaud à Charleville. Étaitil impossible d'être Butor à Paris ?

M. B. - Eh bien, il y a eu un moment, certainement, où il était impossible d'être Butor à Paris et c'est à cause de cela que je suis parti pour l'Égypte. J'écrivais déjà avant de partir pour l'Égypte, j'avais déjà écrit de nombreux poèmes, que je gardais soigneusement pour moi ou que je passais à certains amis quelquefois, et puis j'avais déjà écrit des essais critiques, mais il y a eu un moment où il fallait que je parte (voir Butor, 2008). C'est tout à fait vrai : il y a eu un moment où le Michel Butor à Paris n'était plus possible. Donc la solution a été de partir ; j'ai eu une possibilité de le faire 
dans l'enseignement égyptien et ç’a été un choc considérable. Je n'en suis pas encore revenu complètement. Après, je suis revenu à Paris et je suis allé dans d'autres endroits, et peu à peu, il y a un nouveau Butor qui s'est constitué : un Butor un peu égyptien, un peu anglais, un peu américain, même un peu japonais. Et c'est celui-là qui réussit à survivre, c'est celui-là qui continue d'agir.

C'est à cause de tout cela que je m'efforce de tirer de mon malheur... Bon, je ne peux pas dire que j’ai été spécialement malheureux. Au fond, à regarder de l'extérieur, je n'étais pas plus malheureux qu'un autre. Au contraire, j'étais un enfant qui était né dans une famille très bien, j'étais doué pour diverses choses... Non, j'étais très bien, et pourtant, il y avait une espèce de fissure à l'intérieur. Je sentais de plus en plus qu'il y avait des choses qui n'allaient pas, qui ne marchaient pas. Je me heurtais tout d'un coup à des obstacles que je n'aurais pas imaginés. C'est comme si je marchais dans une rue et puis que, tout d'un coup, un mur tombe au milieu de la rue et que je me tape la tête contre ce mur. Il a fallu que je trouve une solution à tout cela. Pour moi, ç’a été l'écriture et j'ai eu besoin du voyage pour prendre de la distance par rapport à Paris, mais surtout par rapport à celui que j'étais à Paris. Je ne me reprochais rien, ce n'est pas du tout ça, mais il y avait quelque chose qui ne pouvait plus rester tel. Il fallait que je file, il fallait que je mette une distance entre moi et toute ma jeunesse. J'ai passé mon adolescence à Paris sous l'occupation allemande. Là, il y avait une fissure déjà très forte. Ensuite, il y a d'autres fissures qui sont venues et à cette époque-là, je n'aurais certainement pas pu en parler. D'une façon claire. C'est peu à peu qu'il y a des choses qui se sont manifestées, qui se sont éclaircies, et il y a des choses qui ne se sont pas encore éclaircies.

C'est ce qui fait d'ailleurs que je vais peut-être écrire encore. Je n'écrirai plus de romans, ça c'est sûr, je n'écrirai plus non plus de 
gros livres, mais j'écrirai encore beaucoup de petits livres et puis les petits ruisseaux font de grandes rivières ; alors les petits livres les uns à côté des autres, ça finit par faire des bouquins... des tomes d'œuvres complètes comme ça!

R.-M. A. - Restons dans la légèreté : reparlons du suicide. Barthes disait que « la littérature ne peut être à la fois accordée au monde et en avance sur lui, comme il convient à tout art du dépassement, que dans un état de pré-suicide permanent » (Barthes, 1964, p. 69-70) et cela me semble pouvoir s'appliquer à votre œuvre, à la manière dont, systématiquement ou presque, vous vous êtes sabordé vous-même.

M. B. - La formule de Barthes, je l'adopte: une espèce de présuicide permanent. Et ce pré-suicide permanent implique une volonté permanente de résurrection, qui est un thème fondamental de la littérature depuis ses débuts, et bien sûr un thème fondamental du romantisme, avec Chateaubriand qui, parmi ses prénoms, prend le second, René, qui est emblématique. Le thème de la renaissance dans toute la littérature romantique est extraordinairement important. Donc un état de pré-suicide et disons un espoir extraordinaire de renaissance. D'où la constitution, chez certains écrivains, de livres qui sont de véritables matrices, c'est-à-dire de livres qui sont des machines ou des organismes à l'intérieur desquels renaître. Il me semble que c'est tout à fait évident aussi pour Proust, quand il dit qu'il comprend qu'il fallait que son livre ne soit pas seulement comme une cathédrale... Au début, il conçoit son livre comme une cathédrale, ce qui est aussi un thème romantique essentiel - voyez NotreDame de Paris. À un certain moment, il s'aperçoit que son livre ne devrait pas seulement être comme une cathédrale mais comme 
une robe (Proust, 1989, p. 610). Cela veut dire, pour lui, construit avec des morceaux cousus les uns avec les autres. La liaison de ces deux images, cathédrale et robe, c'est quelque chose d'extraordinairement maternel. Le texte est là pour réussir à retrouver le temps, à renaître d'une façon beaucoup plus intéressante. En ce qui me concerne, ce thème de la renaissance était très important ${ }^{5}$. Je ne veux pas simplement me faire renaître moi, je voudrais, si vous voulez - je suis très gourmand -, je voudrais faire renaître tous les autres aussi. Je ne veux pas être rené, je veux participer, même d'une façon infime, à une sorte de renaissance.

R.-M. A. - Jusqu'à essayer de ressusciter les morts ?

M. B. - Ah, évidemment, pour les morts, c'est un problème. Le thème de la mort joue un rôle dans ce que j'ai écrit. Il y a cette idée du suicide positif, qui implique une contemplation de la mort. La mort que l'on peut, dans l'état actuel des choses, la mort que l'on peut retarder, peu à peu, mais qui, de temps en temps, brusquement arrive et souvent d'une façon tout à fait inattendue. Dans tous mes livres, je crois, à l'intérieur du texte, quelque part, il y a un crâne. Vous voyez, c'est le thème de la vanité, dans la peinture ancienne, la nature morte, avec le crâne qui est souvent au milieu des objets qui flattent les sens, donc au milieu de ce qui faisait plaisir dans la vie : au milieu de la nourriture la plus exquise, des bouquets de fleurs, des instruments de musique, des livres, etc. Dans mes livres aussi, il y a un crâne, qui est plus ou moins facile à voir. Quelquefois, il est tout à fait évident, parce que j'en parle, j'emploie le mot; quelquefois je ne l'emploie pas, mais cette méditation sur la mort est toujours présente. J'essaie

5 Il l'est également chez les contemporains de Butor (voir Allemand, 2000, 2001, 2008a, 2008b et 2013). 
bien sûr d'avoir une espèce de fraternité avec les morts.

Tout à l'heure, j'ai parlé des artistes avec qui je travaille et ils peuvent être morts : ça peut aussi bien être un artiste qui vit encore, ce qui est merveilleux, mais ça peut être un artiste qui est déjà mort, et ce que je fais avec lui le ranime en quelque sorte. J'essaie de lui donner la parole et il faut que j'aie le sentiment, lorsque je regarde ses œuvres, qu'il me pardonne, comme la basilique Saint-Marc (voir Butor, 1963), qu'il m'approuve si possible mais qu'au moins, il me pardonne. Il me laisse la liberté de continuer. Alors vous voyez, ça implique une espèce de présence des morts.

Dans mes voyages, j'ai été très impressionné par certains monuments funéraires. L'Égypte, c'est le pays des morts ${ }^{6}$ : la surface occupée par les morts était aussi grande que la surface occupée par les vivants. C'était vrai pour l'Égypte pharaonique mais aussi pour l'Égypte médiévale. À travers l'Islam, ce thème fondamental égyptien de la présence des morts continuait, parce que, au Moyen Âge, dans la ville du Caire, qui était la plus grande du monde méditerranéen, les cimetières étaient plus grands que la ville elle-même. Aujourd'hui, la ville a complètement débordé sur ses propres cimetières, ce qui fait que les fameux tombeaux des califes, que j'ai connus comme un grand cimetière séparé des remparts du Caire par un morceau de désert, eh bien, maintenant, c'est un faubourg et les tombes sont toutes habitées. Les gens se sont installé des maisons entre les tombes, et sur les tombes, et des étages qui montent au-dessus des tombes, et là, il $\mathrm{y}$ a une espèce de cohabitation extraordinaire entre les morts et les vivants.

${ }^{6}$ La mythologie funéraire de l'Égypte antique a beaucoup marqué d'autres écrivains de l'après Seconde Guerre mondiale (cf. Allemand et Roche, 2001, et Allemand et Grainville, 2008). 
Il est certain que dans notre civilisation, le problème de la mort est en quelque sorte escamoté. C'est quelque chose dont on préfère ne pas parler, ce n'est pas du tout mis au centre de notre réflexion, ce n'est pas du tout comme dans certaines autres civilisations. L'Égypte ancienne en est l'exemple par excellence : il y a une relation avec les morts que l'on a perdue un peu en Occident ${ }^{7}$. Il faudrait étudier cela, en particulier autour de ce qui est si important en fait: les monuments aux morts à l'intérieur des cimetières et puis les monuments aux morts de la guerre de 1914. Dans tous les villages français, il y a un monument aux morts de la guerre de 1914. On a dépensé de l'argent pour y graver les noms mais pour la guerre de 1939, il n'y a presque pas de monuments aux morts. On a souvent rajouté quelques noms sur les monuments de la guerre de 1914 ou bien on n'a rien fait du tout, et il y a là un enfoncement de la conscience de la mort. Le fait qu'il y ait eu tellement de gens morts est quelque chose qu'on a recouvert. Et maintenant le fait qu'il y ait tellement de gens qui meurent de façon si horrible dans des quantités de régions du monde, c'est aussi quelque chose que l'on préfère mettre sur le côté de la page de journal.

R.-M. A. - Vous m'avez dit un jour : « La littérature nous aide à regarder nos rêves en face [...]. Et à vivre avec eux. » (Allemand et Butor, 2009, p. 155). Bien sûr, on peut penser à Matières de rêves (Butor, 1975), mais quels sont vos rêves ? Et faites-vous la différence entre rêve et cauchemar?

M. B. - Le mot rêve a plusieurs sens dans notre langue. Il y a d'abord le sens précis : l'activité qui se passe dans le sommeil et

7 Le fait a été développé par Genet dans «L'Étrange mot d'... » à propos de l'urbanisme (voir Allemand, 1992). 
dont nous avons quelquefois souvenir au réveil. Et puis il y a aussi nos rêves, voyez, disons dans la publicité : faites un voyage de rêve, ayez une silhouette de rêve... Pour moi, c'est plus intéressant de $\mathrm{d}^{8} .$. Tout ça, c'est très important d'ailleurs, le rêve dans ce sens-là. Dans mon livre Mobile (1962), j'ai essayé de capter le rêve américain quotidien par l'intermédiaire de la publicité. En étudiant les catalogues des grands magasins, on peut reconstituer la maison dans laquelle l'Américain moyen voudrait vivre. Non pas celle où il vit mais celle dans laquelle il voudrait vivre. La littérature et l'art nous renseignent non seulement sur ce qui est mais aussi sur ce qui n'est pas, c'est-àdire qu'elle nous renseigne sur ce qui nous manque et donc sur nos désirs. C'est très difficile de connaître nos désirs, mais si nous ne réussissons pas à les connaître un peu, ils seront toujours malheureux... Non : ils seront toujours bafoués et donc nous serons obligatoirement malheureux. L'activité nocturne nous permet de connaître un certain nombre de choses en ce qui concerne nos désirs. Il se trouve, je crois, que, malgré la connotation tout à fait positive du mot rêve, la plupart de nos rêves sont en fait des cauchemars, c'est-à-dire qu'il y a un côté désagréable dans nos rêves.

Nous avons quelquefois des rêves magnifiques et très difficiles à raconter. Là aussi, il faut inventer quelque chose pour raconter ces rêves qui nous ont tellement frappés. Vous savez, à la table du petit-déjeuner, dans une famille, quelqu'un dit : "J'ai fait un rêve extraordinaire. » et il essaie d'en faire profiter les autres, et au bout de deux phrases, ça s'est dissipé. Il ne sait plus comment dire; le souvenir de son rêve s'efface. Il y a quelque chose de très positif, qui correspond vraiment à ce que

8 Comme à son habitude, l'écrivain se retient d'être déplaisant (voir Allemand et Butor, 2009. p. 17). 
nous désirons, mais nous sommes extrêmement compliqués, c'est-à-dire que le rêve révèle des problèmes que nous pensions avoir plus ou moins résolus.

Je prends souvent un exemple qui est un des thèmes fondamentaux de Matières de rêves : c'est, chez moi, le rêve de la conférence catastrophique. Je vous ai dit que j'improvisais mes conférences (voir Allemand et Butor, 2010) et j'improvise mes conférences parce que c'est sportif, c'est difficile et ça m'oblige à mobiliser toute mon énergie. Quand j'entre dans une salle de conférence, j'ai bien mes notes, etc., mais j'ai un trac terrible. Chaque fois que j'entrais dans une classe, dans mes cours au lycée aussi bien qu'à l'université, j'avais le trac. Chaque fois. Pourtant, il y avait le public qui était là, l'horloge... Il fallait commencer, donc écarter tout ça et puis plonger, et à partir de ce moment-là, ça commençait à aller, exactement comme un acteur au théâtre. Mais le trac est refoulé, il n'est pas supprimé, et très souvent, la nuit, il se satisfait et j'ai un cauchemar : je fais une conférence qui est catastrophique, le public quitte la salle en me lançant des insultes, etc. C'est bien la réalisation de quelque chose que je craignais, donc la réalisation d'une espèce de désir négatif. Tous les problèmes que nous rencontrons, tout ce que nous avons à refouler se venge, ou se satisfait - mais la vengeance est une satisfaction -, à l'intérieur de nos rêves de la nuit, avec des ramifications, des embranchements d'une complexité extraordinaire.

Donc nos rêves sont très souvent des cauchemars mais ils ont aussi quelque chose d'étonnam... d'extraordinairement ${ }^{9}$ positif.

\footnotetext{
${ }^{9}$ La transcription a conservé le repentir, car il souligne l'emploi insistant de l'adjectif extraordinaire et de son dérivé adverbial, qui font signe vers le rêve baudelairien d'Histoire extraordinaire (Butor, 1961). De même, on aura relevé la récurrence des adverbes évidemment et absolument dans les réponses de l'écrivain.
} 
D'ailleurs, le rêve joue un grand rôle dans l'invention des religions et évidemment, il joue un très grand rôle dans la création artistique. Il y a des exemples célèbres. Un des plus beaux, c'est cette magnifique aquarelle d'Albrecht Dürer, qui dit que c'est le rêve qu'il a fait dans la nuit du tant au tant ${ }^{10}$. Dans la littérature, nous avons des récits de rêves, qui sont quelquefois des récits datés, mais c'est beaucoup plus rare que ce qu'on pourrait croire, parce que le rêve se refuse en quelque sorte au langage. Tous les refoulements qui ont amené à la constitution du rêve nocturne vont se réveiller et c'est ce qui explique la difficulté de raconter le rêve. On commence à raconter et puis quelque chose en nous dit: «Non, c'est ridicule... ça ne vaut plus la peine... je croyais que c'était formidable mais ils ne vont pas comprendre. »Vous voyez, toutes sortes de défenses qui se réveillent à ce moment-là. Alors le rêve nous donne à la fois le Paradis et l'Enfer, et ce qui est important, c'est de réussir à les marier, de faire le mariage du rêve et de l'Enfer. Donc il faut transformer le malheur du rêve en bonheur d'autrui.

R.-M. A. - Je vous remercie.

\begin{abstract}
10 Butor fait référence à l'aquarelle Traumgesicht et à cette note de Dürer : «La nuit du mercredi au jeudi après la Pentecôte [7-8 juin 1525], je vis en rêve ce que représente ce croquis : une multitude de trombes d'eau tombant du ciel. La première frappa la terre à une distance de quatre lieues: la secousse et le bruit furent terrifiants, et toute la région fut inondée. J'en fus si éprouvé que je m'éveillai. Puis, les autres trombes d'eau, effroyables par leur violence et leur nombre, frappèrent la terre, les unes plus loin, d'autres plus près. Et elles tombaient de si haut qu'elles semblaient toutes descendre avec lenteur. Mais, quand la première trombe fut tout près de terre, sa chute devint si rapide et accompagnée d'un tel bruit et d'un tel ouragan que je m'éveillai, tremblant de tous mes membres, et mis très longtemps à me remettre. De sorte qu'une fois levé, j'ai peint ce qu'on voit ci-dessus. Dieu tourne pour le mieux toutes choses. »
\end{abstract}




\section{Bibliographie}

AllEmand, Roger-Michel. 1992, "À propos de "L'Étrange Mot d'..." de Jean Genet » (1987), L'Avant-Livre, Cairon, AmiotLenganey, mars, p. 9-15 ;

—. 2000, "Épurer le langage, se découvrir soi-même », dans Jean-Claude LIEBER et Madeleine RENOUARD (dir.), Le Chantier Robert Pinget, Paris, Jean-Michel Place, p. 105-115;

—. 2001, « Du mythe fondateur à la légende personnelle : l'alchimie selon Butor, Pinget et Robbe-Grillet», dans Chantal FouCrier et Daniel MorTIER (dir.), Pratiques de réécritures. L'autre et le même, Rouen, Publications de l'Université de Rouen, p. 173-188;

—. 2008a, «Patrick Grainville à la recherche de l'unité perdue », @nalyses, vol. 3, n 1 , hiver, p. 1-11;

-. 2008b, «Renouvellement et renaissance dans les Romanesques d'Alain Robbe-Grillet», @nalyses, vol. 3, n² 2, printemps-été, p. 26-50 ;

—. 2011, "Michel Butor ou les cadres du sensible», Phlit, 9 novembre ;

—. 2012, "L'écriture et le silence», dans Roger-Michel Allemand et Márcia ARBEX (dir.), Universo Butor, Belo Horizonte, C/Arte, p. 45-59 ;

- 2013, «Claude Simon pour tout vestige», Babel, $\mathrm{n}^{\circ} 27$, $1^{\text {er }}$ semestre, p. 231-245.

- et Michel Butor. 2009, Michel Butor. Rencontre avec RogerMichel Allemand, Paris, Argol, coll. « Les Singuliers »;

—. 2010, «Michel Butor : propos sur l'enseignement, le jeu et l'improvisation", The French Review, Vol.83, No. 3, February, p. 526-537. 
— et Patrick GRAINVILLE. 2008, " Patrick Grainville, "entre l'aigle pêcheur et le cobra royal” »(1993), @nalyses, vol. 3, n 1, hiver, p. 47-74.

- et Denis Roche. 2001, "L'Aléa littéraire. Entretien sur l'écriture et la photographie" (1993), Ariane, $\mathrm{n}^{\circ} 17$ : "Le Cercle des Muses", Lisbonne, p. 273-302.

BARTHES, Roland. 1964, Essais critiques, Paris, Seuil : « Littérature littérale » (1955), p. 63-70.

BuTOR, Michel. 1961, Histoire extraordinaire, Paris, Gallimard ;

—. 1962, Mobile, Paris, Gallimard ;

-. 1963, Description de San Marco, Paris, Gallimard ;

—. 1975, Matière de rêves, Paris, Gallimard ;

—. 1982, Répertoire $V$, Paris, Minuit : «La littérature et la nuit » (1980), p. 23-32 ;

-. 2008, « Lettres de Michel Butor sur le thème de l'exil, suivies de trois acrostiches inédits", @nalyses, vol. 3, n 1 , hiver, p. 1-20.

Clavel, André et Michel Butor. 1996, Curriculum vitæ. Entretiens avec André Clavel, Paris, Plon.

GENET, Jean. 1968, CEuvres complètes, Paris, Gallimard, coll. «Blanche », t. IV : « L'Étrange Mot d'... » (1967), p. 7-18.

PRousT, Marcel. 1989, À la recherche du temps perdu, t. IV, Le Temps retrouvé (†1927), Jean-Yves TADIE (édit.), Paris, Gallimard, coll. « Bibliothèque de la Pléiade».

RousSEAU, Jean-Jacques. 2009, Les Confessions (†1782 et 1789), Bernard GAGNEBIN et Marcel RAYMOND (édit.), Paris, Gallimard, coll. « Folio classique ». 